\title{
Exploring Attachment and Spirituality among Christians in China
}

\author{
Honghong $\mathrm{Xu}^{1}$, Jenny $\mathrm{Pak}^{2}$, Ruina $\mathrm{Tu}^{2} \&$ Mary Schuberg ${ }^{2}$ \\ ${ }^{1}$ Peking University, Beijing, China \\ ${ }^{2}$ Fuller Theological Seminary, Pasadena, CA, United States \\ Correspondence: Honghong Xu, Peking University, Beijing, China.
}

Received: June 29, 2018

Accepted: July 22, 2018

Online Published: July 30, 2018

doi:10.20849/ajsss.v3i3.410

URL: https://doi.org/10.20849/ajsss.v3i3.410

\begin{abstract}
As research on attachment has exploded, theories and studies on attachment and spirituality also flourished in the west. In contrast, similar researches are scarce in China. Are the attachment theories and measurements applicable universally? Are there any links between parental attachment and God attachment? The present study used mixed methods to examine these questions. First, 355 Christians from Mainland China finished the Relationship Structures Questionnaire (ECR-RS), Attachment to God Inventory, God Image Scales, and Chinese Personality Assessment Inventory-2. Next, a sample of 11 participants were purposefully selected for face-to-face interviews to provide an in-depth understanding of the phenomenon through their life stories and spiritual experiences. Survey results showed significant correlations between parental attachment and spirituality and supported the correspondence theory. Additionally, the higher scores on traditional cultural personality traits correlated with higher scores on insecure attachment. Analysis of themes across 11 cases further revealed patterns between secure/insecure parental attachment and God attachment. Application of western attachment scales in the context of Chinese characteristic parenting styles are discussed.
\end{abstract}

Keywords: attachment, culture, Chinese Christians, spirituality

\section{Introduction}

\subsection{Attachment and Culture}

Research on the parental attachment has exploded in the West since its original work of John Bowlby (1969/1982, 1973) and Mary Ainsworth (Ainsworth \& Bowlby, 1991). They claimed that the attachment styles of the human infant to a caregiver was universal (Ainsworth, Blehar, Waters, \& Wahl, 1978).The western model of secure/insecure attachment theory and experimental paradigm is now widespread in non-western world (Dueck \& Xu, 2017). However, more and more psychologists with cultural sensitivity critique that the Western approach to attachment as nurturing independence is a reflection of a Western "cultural model of virtue" (LeVine \& Norman, 2001, p. 84). They question whether the meaning, nature, and consequences of attachment really remain constant across cultures or not (Dueck \& Xu, 2016). The emerging research suggests that this is not the case (Harwood, Miller, \& Irizarry, 1995; Keller, 2008; LeVine \& Norman, 2001; Otto \& Keller, 2014, Quinn \& Mageo, 2013a; Rothbaum, Weisz et al., 2000; Rothbaum, Morelli, \& Rusk, 2011).Quinn and Mageo (2013) criticize this decontextualized approach to attachment because it misunderstands and sometimes pathologizes attachment styles in non-western cultures. Gaskins (2013) comments that " it is culturally naïve to assign a moral value of 'best' to one point on this spectrum (i.e., secure attachment) when other set points appear to exist in other cultures especially when those differences appear to lead to well functioning and healthy members of those societies" (Dueck \& Xu, 2017).

\subsection{Chinese Culture and Parenting}

According to Oyserman et al.'s (2002) review of 83 studies and meta-analysis to explore the level of individualism and collectivism, they found East Asian countries with Chinese origin are highly collectivistic (Yeo, 2010). Confucianism and Taoism are the core of the Chinese value system; they advocate rejecting individuality and self-assertion, and maintaining the balance between nature, human and spiritual entities. Taoism pays attention to human self-discipline and interpersonal harmony. Confucianism pays more attention to individual fulfillment of social obligations, building interpersonal relationships, obeying social norms, honoring parents and elders, and achieving family honor through personal achievements. These two values have become 
the philosophical basis of Chinese parenting style, which partly determines the expectation and expected response of parent-child interaction. (Chen, 2016; Mchale, Dinh, \& Rao, 2014; Xu, 2005).

Cultural values determine the goals of socialization, affecting the mode of education and practice, and thus related to the development of children (Chao, 2000). Chao's (1995) study, which compared the cultural values of mothers from European American and Asian American backgrounds and their parenting styles, found that the socialization goals of Asian American mothers are consistent with the interdependence and collectivist orientation of Asian culture. Asian American mothers encourage children to achieve high academic achievement and bring honor to the family, thus emphasizing the interdependence of family members. This is different from the goal of socialization in Europe and the United States that emphasizes independence and individualism. European-American mothers are more concerned with the child's self-esteem and the child's happiness as an independent individual (Markus \& Kitayama, 1991; Triandis, 1995).

Parenting style can be evaluated in two dimensions-warmth and control. Generally speaking, American mothers tend to openly express their warmth and intimacy to the child through reasonable control, often praising and promoting the child's independent self-development (Wilcox, 1998). In contrast, Chinese parents emphasize children's compliance and interdependence by emphasizing rules and authority, supporting and satisfying children's needs, and expressing warmth in a more subtle or implicit way (Chao, 1994). Previous research indicated that Asian parenting styles are more authoritarian. Authoritarian parenting styles have been often linked to family conflicts and unhealthy psychological outcomes (e.g., stress, maladaptation, depression, and aggression) in white families; however, in Asian families, it does not lead to negative results but is related to the child's high academic performance.

Some researchers believe that authoritative and authoritarian parenting styles could not fully reflect the whole picture of parenting practice in Chinese culture. $\mathrm{Wu}$ (2002) found that the more common forms of parenting in Chinese tradition, such as teaching, using shame and retrieving love, are related to the authoritative and authoritarian styles in mainland China (Kim \& Wong, 2002). Chao (1994; Chao \& Sue, 1996) has suggested that it is better to use "Guan" or "training" to describe Chinese parenting style. Guan is the use of Chinese traditional beliefs and values to discipline the youngster, at the same time to establish a "supportive, highly involved and physically close mother-child relationship" (Chao, 1994, p. 1112). The strictness in the eyes of Western children might be experienced as control, hostility and distrust, but in Asian children, it is seen as caring and concern.

Agishtein and Brumbaugh's (2013) research showed that Individualism/Collectivism is a potential moderator between culture and adult attachment. For instance, the psychological dependence on others that is integral to collectivist systems points to the possibility of a higher rate of anxious attachment in collectivist cultures (Sorensen \& Oyserman, 2010). Findings from a large-scale cross-cultural study consisting of samples from 62 cultures conducted by Schmitt et al. (2004) also indicated that preoccupied attachment styles were found to be particularly more prevalent in East Asian cultures (i.e., samples from Hong Kong, Taiwan, South Korea, and Japan), and that participants from the East Asian region reported higher scores on the Internal Working Model of Others. Wang and Song (2010) claimed that Chinese adult attachment involves a more complicated cognitive appraising process to identify what is a person's appropriate role and associated status so she or he can act properly, in contrast to spontaneity and authenticity of attachment behaviors reflecting on or guided by one's attachment styles/ needs as emphasized in the Western adult attachment model.

\subsection{Attachment to God}

Granqvist and Kirkpatrick (2013) propose that "God (and other divine figures) function like symbolic attachment figures to whom believers actively strive to obtain or maintain a sense of being connected" (p. 141). The relational bond of love with a transcendent being is said to parallel the affective bond of attachment to a human caregiver. Two general hypotheses have been suggested to describe the connection between parental attachment and God attachment - the compensation hypothesis and the correspondence hypothesis. Previous researches on God attachment have testified those two pathways.

Shin (2009) studied the relationship between parental attachment and the relationship with God in Asian Americans born and raised in the United States. The results showed that participants who had secure attachment with parents also showed security and stability in relationship with God. On the contrary, participants who had insecure parental attachment would also show an insecure and unstable relationship with God. At the same time, those students who were more intimate with their parents showed less disappointment with God and more awareness of God, and desired to worship and serve God more than the insecurely attached students.

Using attachment theory as a conceptual framework, Joung (2006) explored the faith development of ten Korean women in England through a qualitative approach. The study found that participants' image of parents 
corresponded with their image of God. Those who had a positive parental image usually held a similar image of God. Those who had a negative image of parents tended to maintain a negative view toward God. Nevertheless, women who were deprived of parental love sometimes projected an idealized image of God that was opposite to their parental image. Joung (2006) also found that the women's self-image was a reflection of their view of parental care in childhood, and in turn influenced their ways of relating to others and God. Although research on attachment to God flourished in the West, very few similar studies have been conducted in the East. To date, we have not found any research on attachment to God in Mainland China.

\subsection{The Present Studies}

A mixed-study design utilizing quantitative surveys and qualitative interviews were combined to explore the Chinese unique parental attachment and its implications on spirituality. In Study 1, online survey was conducted with 355 Chinese Christians. Parental attachment, God attachment, God image and Chinese traditional personality traits were examined. Since there is no indigenous attachment scale developed yet, we chose three widely used western scales to test their applicability and validity with Chinese population. In Study 2, eleven Christian participants were purposefully selected for the in-depth interviews, which included their narratives of interactions with parents and spiritual experiences throughout their lives. Analysis of these interviews provided the researchers a clear view of cultural specific parent-child relationship dynamics and their subsequent impact on religiosity.

\section{Study 1: Quantitative}

\subsection{Method}

\subsubsection{Participants}

355 participants who were born and raised in Mainland China and converted to Christianity filled out an online survey. Convenient and snowball sampling was used in this study. The recruitment announcement which introduced the study and included the enrollment requirements and informed consent was posted through Wechat to different church workers or pastors. Their participation was voluntary and the online survey was anonymous. The demographic variables are as indicated below. (Table 1)

Table 1. Participant demographics ( $\mathrm{N}=355)$

\begin{tabular}{|c|c|c|c|c|c|}
\hline Variable & & $\mathrm{N}$ & $\%$ & Mean & SD \\
\hline \multicolumn{6}{|l|}{ Gender } \\
\hline & Male & 129 & 36.3 & & \\
\hline & Female & 226 & 63.7 & & \\
\hline \multirow[t]{5}{*}{ Age } & & & & 30.8 & 8.7 \\
\hline & The post $90 \mathrm{~s}$ & 151 & 42.5 & & \\
\hline & The post $80 \mathrm{~s}$ & 136 & 38.3 & & \\
\hline & The post 70s & 44 & 12.4 & & \\
\hline & The post 60 s-older & 24 & 6.8 & & \\
\hline \multicolumn{6}{|l|}{ Education level } \\
\hline & Middle school & 47 & 13.2 & & \\
\hline & High School & 47 & 13.2 & & \\
\hline & Bachelor Degree & 206 & 58 & & \\
\hline & Master or PhD Degree & 55 & 15.5 & & \\
\hline \multicolumn{6}{|l|}{ Marital Status } \\
\hline & Single & 185 & 52.1 & & \\
\hline & Married & 160 & 45.1 & & \\
\hline & Divorced & 10 & 2.8 & & \\
\hline \multicolumn{6}{|l|}{ Church } \\
\hline & House church & 246 & 69.3 & & \\
\hline & Three-self Church & 109 & 30.7 & & \\
\hline Years as Christian & & & & 8.2 & 6.9 \\
\hline \multicolumn{6}{|l|}{ Role in Church } \\
\hline & Normal member & 190 & 53.5 & & \\
\hline & Co-worker & 160 & 45.1 & & \\
\hline & Pastor & 5 & 1.4 & & \\
\hline \multicolumn{6}{|l|}{$\begin{array}{l}\text { Church attendance } \\
\text { frequency }\end{array}$} \\
\hline & Less than once/week & 74 & 20.8 & & \\
\hline & Once/week & 105 & 29.6 & & \\
\hline & Twice/week or more & 176 & 49.6 & & \\
\hline \multicolumn{6}{|l|}{$\begin{array}{l}\text { Parental religious } \\
\text { background }\end{array}$} \\
\hline & $\begin{array}{l}\text { Parents are Christians with great } \\
\text { influence on childrer's conversion }\end{array}$ & 148 & 41.7 & & \\
\hline & $\begin{array}{l}\text { Parents are Christians with no influence } \\
\text { on children's conversion }\end{array}$ & 65 & 18.3 & & \\
\hline & Parents are non-religious & 119 & 33.5 & & \\
\hline & Parents have other religious belief & 23 & 6.5 & & \\
\hline
\end{tabular}




\subsubsection{Instruments}

$E C R-R S$. The Relationship Structures (ECR-RS) questionnaire is a self-report instrument designed to assess attachment patterns in a variety of close relationships. The same 9 items are used to assess attachment styles with respect to two targets (mother; father). The participants were asked to recall their childhood experiences and relationship with mother/father respectively when they filled out the questionnaire. It has two dimension including anxious attachment and avoidant attachment. The test-retest reliability (over 30 days) of the individual scales are approximately .80 in the parental domain (Fraley et al., 2011).

Attachment to God Inventory (AGI). AGI was developed by Beck and McDonald (2004), which is based on the Experiences in Close Relationships (ECR) questionnaire developed by Brennan, Clark, and Shaver (1998). This dimensional measure assesses attachment in terms of Anxiety about Abandonment and Avoidance of Intimacy (Beck \& McDonald, 2004). It has 28-questions and uses a 7-point scale from 1 (disagree strongly) to 7 (agree strongly).

God Image Scales (GIS). GIS contains three dimensions, each dimension has 12 items to measure the Presence ("Is God there for me?"); Challenge ("Does God want me to grow?"); and Acceptance ("Am I good enough for God to love?). The 36 items version was used because of stronger factor structure than 72 items version (Lawrence, 1997).

Chinese Personality Assessment Inventory-2(CPAI-2). CPAI-2 is an indigenous personality scale developed by Cheung et al. (1996). Seven dimensions were used in the present study, including Traditionalism versus Modernity(TM), Renqing(REN), Harmony(HAR), Self versus Social Orientation(SS), Family Orientation(FAM), Face(FACE), and internal versus External Locus of Control(IE).

\subsection{Results}

\subsubsection{Demographic Differences}

Gender differences. There is no significant gender difference on attachment to parents overall; however, male participants had significant higher score on God avoidant and lower score on God presence than female. Male participants also had significant higher score on harmony than female. No gender difference was found on other personality traits.

Group differences. Multi-factor Anova analysis was used to test group differences on attachment and spirituality. First, groups were categorized by parents' religious background. Participants whose conversion experiences were deeply affected by their Christian parents showed more secure mother attachment. They had significant lower score on mother avoidant and mother anxious attachment relationship than other groups. This group also had significant lower scores on Harmony, Family orientation, Renqing and Face. (Table 2)

Table 2. Participants' Spirituality and parents' religious background

\begin{tabular}{|c|c|c|c|c|c|c|}
\hline $\begin{array}{l}\text { Attachment and } \\
\text { Spirituality }\end{array}$ & $\begin{array}{l}\text { Group1 } \\
(\mathrm{n}=148)\end{array}$ & $\begin{array}{c}\text { Group2 } \\
(\mathrm{n}=65)\end{array}$ & $\begin{array}{l}\text { Group3 } \\
(\mathrm{n}=119)\end{array}$ & $\begin{array}{c}\text { Group4 } \\
(n=23)\end{array}$ & $F$ & $P$ \\
\hline $\begin{array}{l}\text { Mother } \\
\text {-Avoidant }\end{array}$ & $19.45 \pm 7.42$ & $23.52 \pm 7.36$ & $24.21 \pm 8.53$ & $21.56 \pm 7.55$ & 9.279 & 0.000 \\
\hline $\begin{array}{l}\text { Mother } \\
\text {-Anxious }\end{array}$ & $5.55 \pm 3.60$ & $7.43 \pm 4.81$ & $6.66 \pm 4.23$ & $8.00 \pm 5.86$ & 4.514 & 0.004 \\
\hline $\begin{array}{l}\text { Father } \\
\text {-Avoidant }\end{array}$ & $23.42 \pm 7.98$ & $25.38 \pm 9.00$ & $24.17 \pm 7.88$ & $27.00 \pm 10.16$ & 1.737 & 0.159 \\
\hline $\begin{array}{l}\text { Father } \\
\text {-Anxious }\end{array}$ & $6.04 \pm 4.35$ & $7.23 \pm 5.09$ & $7.04 \pm 4.55$ & $7.82 \pm 5.89$ & 1.922 & 0.126 \\
\hline $\begin{array}{l}\text { God } \\
\text {-Avoidant }\end{array}$ & $42.49 \pm 10.89$ & $42.43 \pm 12.08$ & $42.61 \pm 12.49$ & $41.13 \pm 11.38$ & 0.105 & 0.957 \\
\hline $\begin{array}{l}\text { God } \\
\text {-Anxious }\end{array}$ & $45.28 \pm 16.65$ & $45.12 \pm 16.52$ & $44.35 \pm 17.11$ & $47.30 \pm 17.51$ & 0.215 & 0.886 \\
\hline God Acceptance & $39.04 \pm 4.83$ & $38.72 \pm 5.20$ & $38.15 \pm 6.24$ & $38.52 \pm 6.40$ & 0.580 & 0.628 \\
\hline God Challenge & $33.65 \pm 3.24$ & $33.40 \pm 3.39$ & $33.65 \pm 3.60$ & $33.95 \pm 3.63$ & 0.173 & 0.915 \\
\hline God Presence & $38.43 \pm 4.95$ & $37.70 \pm 6.09$ & $36.60 \pm 5.86$ & $37.21 \pm 5.25$ & 2.464 & 0.062 \\
\hline
\end{tabular}

Group1: Parents are Christians with great influence on children's conversion

Group2: Parents are Christians with no influence on children's conversion

Group3: Parents are non-religious

Group4: Parents have other religious belief 
Secondly, based on the role of church involvement, three groups were categorized: regular church member, church coworker, and pastor. Church co-workers and pastors scored lower on insecure attachment with God, and scored higher on positive God image, including God acceptance and presence. These differences had statistical significance. (Table 3)

Table 3. Participants' spirituality and church roles

\begin{tabular}{llllll}
\hline $\begin{array}{l}\text { God Attachment } \\
\text { and God Image }\end{array}$ & $\begin{array}{l}\text { Normal member } \\
(\mathrm{N}=190)\end{array}$ & Coworker & Pastor & F & P \\
\hline God -Avoidant & $44.66 \pm 11.98$ & $40.06 \pm 10.78$ & $33.60 \pm 7.53$ & 8.588 & 0.000 \\
God & $46.49 \pm 16.16$ & $43.83 \pm 17.38$ & $31.20 \pm 13.93$ & 2.856 & 0.059 \\
-Anxious & & & & & \\
God Acceptance & $37.39 \pm 5.36$ & $40.01 \pm 5.33$ & $42.60 \pm 4.44$ & 11.864 & 0.000 \\
God Challenge & $33.39 \pm 3.41$ & $33.88 \pm 3.38$ & $34.40 \pm 3.84$ & 1.014 & 0.364 \\
God Presence & $36.55 \pm 5.63$ & $38.74 \pm 5.23$ & $41.40 \pm 3.20$ & 8.298 & 0.000 \\
\hline
\end{tabular}

Although this study differentiated Three-Self Church and House Church participants, no significant difference was found between these two groups.

Correlations among parental attachment, God attachment and God image. Parental attachment showed significant correlation with God attachment; more specifically, anxious parental attachment positively correlated with insecure God attachment, and negatively correlated with God presence and God acceptance. (Table 4)

Table 4. Participants' spirituality and parental attachment

\begin{tabular}{|c|c|c|c|c|c|c|c|c|c|}
\hline $\begin{array}{l}\text { Attachmen } \\
\mathrm{t} \quad \text { and } \\
\text { Spirituality }\end{array}$ & $\begin{array}{l}\text { Mother- } \\
\text { Avoidan } \\
\mathrm{t}\end{array}$ & $\begin{array}{l}\text { Mother } \\
\text { Anxious }\end{array}$ & $\begin{array}{l}\text { Father- } \\
\text { Avoidan } \\
t\end{array}$ & $\begin{array}{l}\text { Father- } \\
\text { Anxiou } \\
\text { s }\end{array}$ & $\begin{array}{l}\text { God } \\
- \\
\text { Avoidan } \\
\mathrm{t}\end{array}$ & $\begin{array}{l}\text { God } \\
- \\
\text { Anxiou } \\
\text { s }\end{array}$ & $\begin{array}{l}\text { Acceptanc } \\
\text { e }\end{array}$ & $\begin{array}{l}\text { Challeng } \\
\mathrm{e}\end{array}$ & $\begin{array}{l}\text { Presenc } \\
\mathrm{e}\end{array}$ \\
\hline $\begin{array}{l}\text { Mother } \\
\text {-Avoidant }\end{array}$ & 1 & $.233^{* *}$ & $.341^{* *}$ & .099 & .056 & .097 & $-.106^{*}$ & .008 & -.075 \\
\hline $\begin{array}{l}\text { Mother } \\
\text {-Anxious }\end{array}$ & $.233^{* *}$ & 1 & .098 & $.575^{* *}$ & $.196^{* *}$ & $.227^{* *}$ & $-.264^{* *}$ & $-.126 *$ & $-.195^{* *}$ \\
\hline $\begin{array}{l}\text { Father } \\
\text {-Avoidant }\end{array}$ & $.341^{* *}$ & .098 & 1 & $.250^{* * *}$ & .023 & .097 & -.034 & -.059 & -.073 \\
\hline $\begin{array}{l}\text { Father } \\
\text {-Anxious }\end{array}$ & .099 & $.575^{* *}$ & $.250^{* *}$ & 1 & $.155^{* * *}$ & $.249 * *$ & $-.264^{* *}$ & -.104 & $-.193^{* *}$ \\
\hline $\begin{array}{l}\text { God } \\
\text {-Avoidant }\end{array}$ & .056 & $.196^{* *}$ & .023 & $.155^{* *}$ & 1 & $.428^{* *}$ & $-.549^{* *}$ & $-.211^{* *}$ & $-.656^{* *}$ \\
\hline $\begin{array}{l}\text { God } \\
\text {-Anxious }\end{array}$ & .097 & $.227^{* *}$ & .097 & $.249 * *$ & $.428^{* *}$ & 1 & $-.606^{* *}$ & $-.135^{*}$ & $-.415^{* *}$ \\
\hline Acceptance & $-.106^{*}$ & $-.264^{* *}$ & -.034 & $\begin{array}{l}-.264^{*} \\
*\end{array}$ & $-.549 * *$ & $\begin{array}{l}-.605^{*} \\
*\end{array}$ & 1 & $.339 * *$ & $.732^{* *}$ \\
\hline Challenge & .008 & $-.126^{*}$ & -.059 &.- .104 & $-.211^{* *}$ & $-.135^{*}$ & $.339 * *$ & 1 & $.325^{* *}$ \\
\hline Presence & -.075 & $-.195 * *$ & -.073 & $-.193^{*}$ & $-.656^{* *}$ & $-.415^{*}$ & $.732^{* *}$ & $.325 * *$ & 1 \\
\hline
\end{tabular}

Parental attachment also related to cultural characteristic personality traits. This study showed that avoidant attachment with mother has significant positive correlation with harmony, family orientation, traditionalism, Renqing and face; anxious attachment with mother had significant positive correlation with harmony and family orientation and negative correlation with self orientation. On the other hand, avoidant attachment with father had significant positive correlation with harmony, family orientation, traditionalism and face; anxious attachment with father had significant positive correlation with harmony, family orientation, Renqing and internal locus of control. (Table 5) 
Table 5. Participants' parental attachment and traditional personality traits

\begin{tabular}{|c|c|c|c|c|c|c|c|c|c|c|c|}
\hline $\begin{array}{l}\text { Attachment } \\
\text { to Parents } \\
\text { Personality }\end{array}$ & $\begin{array}{l}\text { Mother } \\
\text { and- } \\
\text { Avoidant }\end{array}$ & $\begin{array}{l}\text { Mother } \\
- \\
\text { tAnxious }\end{array}$ & $\begin{array}{l}\text { Father } \\
\text { - }\end{array}$ & $\begin{array}{l}\text { Father } \\
\text { t Anxious }\end{array}$ & S HAR & FAM & TM & REN & SS & IE & FACE \\
\hline $\begin{array}{l}\text { Mother } \\
\text {-Avoidant }\end{array}$ & 1 & $.233^{\circ}$ & $.341^{\circ}$ & .099 & $.198^{\circ *}$ & " & .139 & ". & ${ }^{\circ}-.140^{\circ}$ & ”.091 & $.363^{\circ}$ \\
\hline $\begin{array}{l}\text { Mother } \\
\text {-Anxious }\end{array}$ & $.233^{\circ}$ & 1 & .098 & $.575^{\circ}$ & $.245^{\circ}$ & $.143^{*}$ & -.083 & .067 & $-.177^{\circ}$ & “.069 & .067 \\
\hline $\begin{array}{l}\text { Father } \\
\text {-Avoidant }\end{array}$ & $.341 *$ & .098 & 1 & $.250^{\circ}$ & $.148^{\circ}$ & ${ }^{*} .377^{*}$ & $.123^{\circ}$ & .101 & -.099 & -.001 & $.220^{\circ}$ \\
\hline $\begin{array}{l}\text { Father } \\
\text {-Anxious }\end{array}$ & .099 & $.575^{\circ}$ & $.250^{\circ}$ & 1 & $.222^{*}$ & " $178^{\circ}$ & -.054 & $.119^{\circ}$ & -.098 & $.115^{\circ}$ & .040 \\
\hline HAR & $.198^{\circ "}$ & $.245^{\circ}$ & $.148^{\circ}$ & $.222^{\circ}$ & 1 & $.424^{\circ}$ & .074 & $.226^{*}$ & $-.221^{\circ}$ & "..091 & $.190^{\circ}$ \\
\hline FAM & $.409^{*}$ & $.143^{*}$ & $.377^{\circ}$ & $.178^{*}$ & $.424^{*}$ & 1 & .031 & .047 & $-.294^{\circ}$ & $" .210 "$ & $.569^{\circ}$ \\
\hline TM & $.139^{*}$ & -.083 & $.123^{\prime}$ & -.054 & .074 & .031 & 1 & $.412^{* *}$ & $.105^{\circ}$ & $-.143^{*}$ & .228 \\
\hline REN & $.151^{\circ}$ & .067 & .101 & $.119^{\circ}$ & $.226^{\circ}$ & .047 & $.412^{*}$ & 1 & .091 & $-.145^{*}$ & $.260^{\circ}$ \\
\hline SS & $-140^{\circ "}$ & $-.177^{*}$ & -.099 & -.098 & $-.221^{\circ}$ & $"-294^{*}$ & $.105^{\circ}$ & .091 & 1 & $-.187^{\circ}$ & -1.147 \\
\hline $\begin{array}{l}\text { IE } \\
\text { FACE }\end{array}$ & $\begin{array}{l}.091 \\
.363^{*}\end{array}$ & $\begin{array}{l}.069 \\
.067\end{array}$ & $\begin{array}{l}-.001 \\
.220\end{array}$ & $\begin{array}{l}.115^{\circ} \\
.040\end{array}$ & $\begin{array}{l}.091 \\
.190\end{array}$ & $\begin{array}{l}.210^{\circ} \\
.569^{\circ}\end{array}$ & $\begin{array}{l}-.143^{\circ} \\
.228^{\circ}\end{array}$ & $\begin{array}{l}-145^{*} \\
.260 "\end{array}$ & $-187^{\circ}$ & $\ddot{*}_{-.003}^{1}$ & $\begin{array}{l}-.003 \\
1\end{array}$ \\
\hline $\begin{array}{l}{ }^{* *} \text {. Correlatio } \\
{ }^{*} \text {. Correlation }\end{array}$ & $\begin{array}{l}\text { ificant at } t \\
\text { ficant at th }\end{array}$ & $\begin{array}{l}\text { he } 0.01 \mathrm{le} \\
\text { e } 0.05 \mathrm{lev}\end{array}$ & $\begin{array}{l}\text { evel (2-ta } \\
\text { vel (2-tai }\end{array}$ & & & & & & & & \\
\hline
\end{tabular}

Spirituality correlates with personality traits. Avoidant attachment with God positively related with harmony, negatively related with self-orientation;

anxious attachment with God positively related with harmony, family orientation and internal locus of control, negatively related with traditionalism and self-orientation.

Harmony, family orientation and internal locus of control had significant negative correlations with God acceptance and God presence. In addition, insecure God attachment appeared to predict negative God image. Anxious/Avoidant attachment to God had significant negative correlations with God acceptance and presence.

Table 6. Participants' spirituality and personality

\begin{tabular}{|c|c|c|c|c|c|c|c|c|c|c|c|c|}
\hline Spirituality and & God - & God. & & & & & & & & & & \\
\hline Personality & avoidant & Anxious & Acceptan & ceChalleng & gePresence & SHAR & FAM T & TM & REN & SS & IE & FACE \\
\hline $\begin{array}{l}\text { God } \\
\text {-avoidant }\end{array}$ & 1 & $.428^{\circ}$ & $-.549^{*}$ & $-.211 "$ & $-.656^{*}$ & $.297^{\circ \prime}$ & .084 & -.015 & .048 & $-.132^{\circ}$ & .030 & -.070 \\
\hline God-Anxious & $.428^{\circ}$ & 1 & $-.606^{*}$ & $-.135^{\circ}$ & $-.415^{*}$ & $.355^{\circ}$ & $323^{*}$ & $-.113^{\circ}$ & .009 & $-.137^{\circ}$ & $.192^{*}$ & -.064 \\
\hline Acceptance & $-.549^{*}$ & $-.606^{*}$ & 1 & $.339^{*}$ & $.732^{*}$ & $-350^{\circ}$ & $-230^{\circ}$ & .061 & .006 & $.149^{\circ "}$ & $"-.151 "$ & $" .028$ \\
\hline Challenge & $-.211^{*}$ & $-.135^{\circ}$ & $.339^{*}$ & 1 & $.325^{\circ}$ & $-167^{*}$ & -101 & .063 & .047 & .038 & $-.144^{*}$ & "-.068 \\
\hline Presence & $-.656^{*}$ & $-.415^{\circ}$ & $.732^{\circ}$ & $.325^{*}$ & 1 & $-306^{*}$ & $\cdot .154^{*}$ & -023 & -.082 & .082 & $-.113^{\circ}$ & 3.029 \\
\hline HAR & $.297^{*}$ & $.355^{*}$ & $-.350^{*}$ & $-.167^{*}$ & $-.306^{*}$ & 1 & $.424^{*}$ & .074 & $.226^{*}$ & $-221 "$ & “.091 & $.190^{\circ}$ \\
\hline FAM & .084 & $.323^{*}$ & 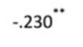 & -.101 & $-.154 *$ & $.424^{\circ}$ & 1 & .031 & .047 & $-294^{*}$ & ${ }^{*} .210$ & $.569^{*}$ \\
\hline TM & -.015 & $-.113^{\circ}$ & .061 & .063 & -.023 & .074 & .031 & 1 & $.412^{*}$ & $.105^{\circ}$ & $-.143^{*}$ & 3.228 \\
\hline REN & .048 & .009 & .006 & .047 & -.082 & $.226^{\circ}$ & .047 & $.412^{*}$ & 1 & .091 & $-.145^{*}$ & $" .260 "$ \\
\hline Ss & $-.132^{\circ}$ & $-.137^{\circ}$ & $.149^{*}$ & .038 & .082 & $-221^{\prime \prime}$ & $" .294 "$. & $.105^{\circ}$ & .091 & 1 & $-.187^{*}$ & ". \\
\hline IE & .030 & $.192^{*}$ & $-.151{ }^{*}$ & $-.144^{*}$ & $-.113^{\circ}$ & .091 & $.210^{*}$ & -.143 & $\because .145 "$ & ${ }^{*}-187$ & & -.003 \\
\hline FACE & -.070 & -.064 & .028 & -.068 & .029 & $.190^{\circ}$ & $.569^{\circ}$ & $.228^{*}$ & $.260^{\circ}$ & $"-147^{*}$ & "-.003 & 1 \\
\hline
\end{tabular}

\section{Study 2: Qualitative}

\subsection{Method}

\subsubsection{Participants}

After obtaining the approval for the study from the Institutional Review Board, in-depth interviews were carried out. The respondents ranged in age from 21 to 70 years (mean $=38.4$ years). The majority $(81 \%)$ of the respondents had Bachelor's degree. Potential participants were identified by reference of pastors and friends. 
The sample of potential participants was purposively drawn to include men and women with different levels of education, income, different SES of original family, and years since conversion. A total of 11 participants were selected for the interview. (Table 7)

Table7. Participant demographics

\begin{tabular}{|c|c|c|c|c|c|c|c|c|c|c|}
\hline & $\begin{array}{l}\mathrm{M} / \\
\mathrm{F}\end{array}$ & $\begin{array}{l}\mathrm{Ag} \\
\mathrm{e}\end{array}$ & $\begin{array}{l}\text { Marit } \\
\text { al } \\
\text { status }\end{array}$ & $\begin{array}{l}\text { Years } \\
\text { after } \\
\text { baptis } \\
\text { m }\end{array}$ & $\begin{array}{l}\text { Educati } \\
\text { on }\end{array}$ & $\begin{array}{l}\text { Occupation } \\
\text { Self }\end{array}$ & $\begin{array}{l}\text { Occupation } \\
\text { Father }\end{array}$ & $\begin{array}{l}\text { Occupation } \\
\text { Mother }\end{array}$ & $\begin{array}{l}\text { Primary } \\
\text { Caretaker }\end{array}$ & $\begin{array}{l}\text { Childho } \\
\text { od } \\
\text { Residen } \\
\text { ce }\end{array}$ \\
\hline P1 & $\mathrm{F}$ & 34 & $\begin{array}{l}\text { marri } \\
\text { ed }\end{array}$ & 9 & phd & $\begin{array}{l}\text { Univesity } \\
\text { teacher }\end{array}$ & $\begin{array}{l}\text { Principle } \\
\text { primary } \\
\text { school }\end{array}$ & Accountant & $\begin{array}{l}\text { grandma until } \\
\text { 5y.o;then } \\
\text { parents }\end{array}$ & Village \\
\hline P2 & $\mathrm{F}$ & 49 & $\begin{array}{l}\text { marri } \\
\text { ed }\end{array}$ & 8 & BS & Teacher & worker & $\begin{array}{l}\text { Principle } \\
\text { primary school }\end{array}$ & parents & city \\
\hline P3 & $\mathrm{F}$ & 70 & $\begin{array}{l}\text { marri } \\
\text { ed }\end{array}$ & 15 & $\begin{array}{l}\text { middle } \\
\text { school }\end{array}$ & $\begin{array}{l}\text { manager of } \\
\text { bank, retired }\end{array}$ & merchant & housewife & parents & city \\
\hline P4 & $\mathrm{F}$ & 39 & single & 3 & BS & $\begin{array}{l}\text { sales work in } \\
\text { IT company }\end{array}$ & $\begin{array}{l}\text { Senior } \\
\text { engineer } \\
\text { and leader } \\
\text { in almine } \\
\text { coalmine } \\
\text { group }\end{array}$ & housewife & parents & Town \\
\hline P5 & $\mathrm{F}$ & 48 & $\begin{array}{l}\text { marri } \\
\text { ed }\end{array}$ & 7 & BS & $\begin{array}{l}\text { Teacher; Ther } \\
\text { apist }\end{array}$ & $\begin{array}{l}\text { engineer in } \\
\text { an arsenal }\end{array}$ & $\begin{array}{l}\text { athlete, then } \\
\text { worker }\end{array}$ & parents & city \\
\hline P6 & M & 48 & $\begin{array}{l}\text { marri } \\
\text { ed }\end{array}$ & 15 & MBA & $\begin{array}{l}\text { Techinical } \\
\text { Manager }\end{array}$ & Journalist & Journalist & $\begin{array}{l}\text { parents; } 10 \mathrm{mo} \\
\text { nths-2years } \\
\text { old,grandpare } \\
\text { nts }\end{array}$ & City \\
\hline P7 & M & 24 & single & 2 & BS & Engineer & $\begin{array}{l}\text { businessma } \\
n\end{array}$ & housewife & parents & Village \\
\hline P8 & M & 30 & single & 2 & BS & IT engineer & farmer & $\begin{array}{l}\text { farmer; housewi } \\
\text { fe;no education }\end{array}$ & parents & Village \\
\hline P9 & $M$ & 28 & single & 2 & BS & engineer & worker & housewife & parents & Village \\
\hline $\begin{array}{l}\mathrm{P} 1 \\
0\end{array}$ & M & 31 & single & 4 & BS & IT engineer & $\begin{array}{l}\text { driver, insura } \\
\text { nce saleman }\end{array}$ & $\begin{array}{l}\text { worker;housewi } \\
\text { fe }\end{array}$ & parents & Village \\
\hline $\begin{array}{l}\mathrm{P}_{1} \\
1\end{array}$ & $\mathrm{~F}$ & 21 & single & 2 & $\begin{array}{l}\text { middle } \\
\text { school }\end{array}$ & waitress & farmer & $\begin{array}{l}\text { farmer, housewi } \\
\text { fe }\end{array}$ & $\begin{array}{l}\text { raised by } \\
\text { grandparents }\end{array}$ & Village \\
\hline
\end{tabular}

\subsubsection{Procedure}

Interview format. To obtain information that was culturally specific, respondents were asked to discuss their attachment relationship with primary care givers (e.g., mother, father, grandparents, etc.), before and after their religious conversion and their relationship with God. Specifically, the participants were asked to describe the characteristics of interactions with their parents. These questions were augmented with additional queries to explore indigenous dimensions of attachment to parents and God that have not been previously uncovered by Western research. Based on Granqvist's (1998) work on God attachment, respondents were also asked to describe the ways in which they related to God.

Analytic strategy. The individual interviews were tape recorded, transcribed into Chinese, and subsequently translated into English. Qualitative data analysis was conducted simultaneously by English (by one of the authors and two graduate research assistants; Team A) and in Chinese (by the other two authors and a graduate research assistant; Team B). Our coding schema was developed both inductively and deductively through a series of steps. First, each of the authors read a subset of the transcripts independently to develop a preliminary coding schema. Second, we reviewed each other's schemas and added codes that had been suggested by previous research on parental attachment and God attachment in the United States and China. Third, we applied the resulting coding framework to the data. Finally, we compared the qualitative data that emerged within each category in both the Chinese and English translations. We identified the conceptual overlap within the data and further consolidated the coding categories. After the initial reading and coding, the research team A met to discuss each case in depth, to review the coding of all 11 cases, and to resolve any discrepancies in interpreting passages from the interviews and in coding. Subsequently, Team A and Team B held conference calls to further discuss and review the codes, and resolve any discrepancies in interpreting passages and coding. The researchers took memos of insights that evolved out of these discussions. Based on these insights, codes were grouped in coordinating themes and finally in a more conceptual matrix that aimed at discerning trends across cases.

Because this study was conducted in the major city, findings from this study might not readily transfer to rural areas. Nevertheless, it is believed that experiences and perceptions of young adults in urban areas would not be 
entirely disconnected from the rest of the country given the profound effects of core traditional cultural values and relational practices rooted in China (Sundararajan, 2015).

\subsection{Results}

Table8. Themes and examples

\begin{tabular}{lll}
\hline & Themes & Participants \\
\hline Father & Secure Attachment & \\
\hline Mother & Quality time, dependable, warm, economic provision & P3, P6 \\
\hline God & Chong, Teng, Guan* & P1, P3, P5, P9, P10 \\
\hline & Savior, unconditional love & P2, P3, P4, P5 \\
\hline Father & Insecure Attachment & \\
\hline Mother & Absent, distant, harsh, punitive & P1, P2, P4, P5, P7, P8, P9, P11 \\
\hline God & Judgmental, absent, distant & P2, P4, P6, P7, P8, P11 \\
\hline
\end{tabular}

$*$ Chong $=$ indulging; Teng $=$ heart-aching love; Guan $=$ loving but firm guidance

\subsubsection{Relationship with Mother}

\subsubsection{Secure Attachment}

'Chong'(宠) and 'Teng' (疼). Participants with secure attachment tended to report happy memories when describing their relationship with their mothers during childhood. The mother's affectionate love became the basis of secure parental attachment. Five out of 11 interviewees have been identified with secure attachment to their mothers. Three of them (P1, P3, P5) used 'chong' (doted on) and 'teng' (heart-aching love) to describe mother's love. 'Chong' means indulge with every attention. Children with 'chong' would feel special and loved by their parents. This kind of love can be manifested in many ways such as physical touch (e.g., hugs, kisses, etc.).

One of the participants explained her love language as a high frequency of physical touch. She described a very intimate relationship with her mother when she was young which manifested as hugging frequently and sharing the same bed. However, physical touch was not common for other participants in their recollections of interactions with their mothers which also aligns with the overall cultural tendency in China. Parents may physically express their affection to babies under 3 years old because they assume babies do not understand orders. However, parents decrease more overt physical expression when children turn 4 or 5years old, and begin to discipline them by directive words.

Another expression of 'chong' may be found in the special treatment of a child by way of giving him/her special treats such as candy or toy. This form may be very obvious in a family with siblings. For example, Participant 3 stated,

We were very poor at that time. Life was very difficult. My mother often gave me snacks secretly. My brothers are 7 years and 2 years older than me. Whenever they were naughty, my mother would praise me. She said, 'Look at your little sister, how good is she! Look at yourselves! Too bad!'

Another example of this form of 'chong' was offered by Participant 5:

One day my little brother found an egg which was just laid by neighbor's chicken. He brought it home and gave it to our mother gladly. Then he went outside to play. My mother gave this fresh egg to me secretly and whispered to me, 'eat it now, it is fresh and good for your health.'

'Teng' is another common type of love expressed by Chinese parents. The difference between 'teng' and 'chong' is that 'teng' is out of concern and worry. It is referred to as the heart-aching love. Mother cares particularly about the physical well-being of her child and worries about whether or not the child has eaten well or is warm enough. For example, Participant 5 reported that when she would get sick her mother would be very anxious and subsequently give her many medicines. Participant 1 also recalled how her "mother thought school meals were 
not good enough, so she prepared lunch, rode bike to give [her] the perfect lunch. [Her] classmates in [her] dorm were very envious."

'Teng' toward parents ---how children keep parents worry free. According to attachment theory, parents as an attachment figure could serve as a secure base for children when they get upset. This idea was explored during the interview by asking the participants to describe who they generally turn to when they have trouble, feel upset, or are wronged. Nearly every interviewee answered that they would deal with it by themselves or talk with classmates or friends. They were more inclined to keep their struggle from their parents because they did not want to make parents worry. Participant 1 explained:

I did not want her to worry too much. So if I had problems at school, I would choose to solve by myself. It became my habit. I still remember when I was in middle school, my English teacher did not like me very much. He always gave me hard times and made me very embarrassed in front of the class. He scolded me and punished me. But I never told my mother about this. I felt ashamed. I knew if I told her, she would be very upset. Now I am an adult, I still keep this habit--only report good news to her, not bad news at all.

Gentle "guan jiao" (discipline). In China the mother bears the primary responsibility for the discipline of children. Different discipline styles have variable influences on types of attachment bonding that occurs between child and mother. Gentle and patient "guan jiao" on the one hand, could be an important source of love that children implicitly recognize; on the other hand, harsh, punitive and shame-centered "guan jiao," which could be read by the child as resentment and aversion.

Moral/behavioral training'. One of the participants (P9) with a mother who is a Christian described himself as very obedient since a young age, recalling how his mother would firmly correct him whenever he would make a mistake. His mother disciplined her three boys for good behaviors and warned them against stealing, robbing, cheating, bullying and lying. As they grew up, their mother became more tolerant. She paid increasing attention to their feelings out of concern to save their face.

Attitudes toward school education. There was a great emphasis on education by the mothers of most of the interviewees and these mothers would go to great lengths to aid in their studying. Participant 5 talked about her conflicts with teachers and students when she was in middle school. She felt aggravated and insulted at school. Her mother showed sensitivity and awareness of the participant's experience by helping her transfer to another school. Participant 9 also recalled an important event in his life for which he still felt grateful to his mother for the way she intervened. He was about to drop out of school due to his family's poor economic situation and his father suggested quitting school; however, his mother insisted that he must continue and borrowed money from relatives in order to cover for his school tuition. Participant 9 now graduated from college and works as an engineer. With the persistence of his mother, all three sons from the impoverished family background managed to attend college.

\subsubsection{Insecure Attachment}

Six interviewees were identified as having insecure attachment with their mothers. Three common factors were found related to this attachment style.

Strict authoritarian control. In the present study, we found that extreme authoritarian parenting as indicated by three participants contributed to unhappy childhood memories and a lack of emotional security. For example, Participant 4 stated, "My mother was so dominating. She only cared about my education. Except study, I should not think about anything else." He also described, "She was furious and bad tempered. I remember. Once, when I was little, she had an emotional outburst. She kicked me very hard. We were at a hill at that time. She kicked me and I rolled down from the hill." Participant 2 also indicated:

My parents punished me physically. I was scared. I was just a little girl. They should not do this to me. My mother beat me. So did my father. When they did this a lot to me, I felt oppressed. I was very upset. I wanted to grow up fast and leave them forever. But when I grew up, I realized that they did this for my own good.

Emotional neglect or abuse. A lack of response to a child's physical and emotional needs will lead to insecure attachment bonding according to attachment theory. The caregiver may be physically present but emotionally unavailable to the child. Participant 4's mother had bipolar disorder and later diagnosed with schizophrenia. Her mother demonstrated a pattern of "neglect" and was unable to provide adequate support. Mother could say she loved her daughter, but she was not able to anticipate or perceive her daughter's needs. Participant 4 explained, "She was cold and distant. She ignored me and shut herself up with books. She loved reading. There were a lot of books in my home. My mother immersed herself in the world of books." Participant 7 also described her experience of her mother in the following ways: 
She was bad and quick tempered. In fact, she wasn't a gentle woman. She did not show her love and understanding for my father. For me, she was very selfish. She put all her wish and expectation on me. Sometimes, her love for me was deformed.

Unstable family environment. It has been said that the best gift you can give to your child is your own happy marriage. If parents continually fight with each other the child is deprived of feeling emotional security. Harmonious family atmosphere is essential to the healthy formation of secure attachment bonding. Three participants talked about painful memories of their parents' chronic conflicts during childhood. Participant 4 explained, "I remember my parents were quarrelling all the time, and we seldom sat together to have meals. My family was always cold, dark and quiet. It was like no one was living at home." Participant 7" also described, "My mother was not in good relationship with my father and she always told me that she didn't divorce my father only because of me." Participant 8 similarly indicated, "They were always fighting each other. I still remember when I was 8 or 9 years old, I held my two younger sisters' hands, hid in bed, covered our heads with blanket, and still could hear their quarrelling outside."

\subsubsection{Relationship with Father}

This study showed that fathers in China are not very involved in parenting. Interviewees only spent a third of time allotted to describe relationship with their fathers. The father-child relationship appeared more distant than mother-child relationship. Even so, three participants still captured several warm moments being with their fathers.

\subsubsection{Secure Attachment}

Quality time. When Participant 3 talked about her father, she could not hide her great excitement. She confidently described how her father showed fondness for her. She still remembered how she went on a business trip with him 65 years ago.

Good enough substitute caregiver when mother is not available. Although in China the father is not actively involved in child rearing, he could serve as substitute caregiver and establish secure bonding with children if the mother is not available due to sickness or other reasons. Despite this limited time and potential for intimate moments, the father-child relationship could nevertheless help build a foundation of security and unforgettable memories. For example, Participant 7 indicated the following:

When I was 7, my father took me to his hometown. It was really great. I thought my father was a good father. It was in winter. You know the south in the winter was very cold. My father warmed the bedding for me every night. I would be frozen to death without my father. (laugh...) It was like ice cave there.

\subsubsection{Insecure Attachment}

Just as with the mother-child attachment, father's authoritarian control could also contribute to insecure bonding. For instance, Participant 2 explained:

My father was 'old revolutionary.' He had strict rules at home, such as I had to comb my hair together. I felt very restricted. I remember one thing, I lost my umbrella, they criticized me. I argued with them. I felt so angry, so upset. So I ran away from home. My mother was anxious and finally got me back. I cried heavily. As a child, I felt they did not love me. Why they were so strict? I had a lot of emotions in my heart but nowhere to express. But when I saw my mother was also worried about me, so, I thought they might love me.

Participant 7 also described:

When I was little, I cried very easily. I was weak in my character. For example, if I had a fight with other kids, I could not win them. I lost and cried. And when I got home, my father criticized me, "Why did you not win the fight, even you are fighting with a younger kid than you?" He criticized me harshly. He did not hit me for this, but I still felt hurt deeply. I would rather be hit by him.

\subsubsection{Relationship with God}

Born and raised in an atheistic environment, many participants did not have opportunity to learn about God and Christianity or to actively explore spirituality. Based on the interviews, several factors were related to the conversion experience.

Doubting previous worldviews and searching for meaning of life.

Participant 9 shared his experiences in the following way: "To be honest, born and growing up in China, students and citizens, many things such as education, are controlled. We cannot get to know many things, including 
religion and faith. When I started to get to know Christian culture, I realized what I learned before, such as life meaning, human being, love, etc., are totally limited, even bullshit. I was cheated for so many years."

Christian role model and Christian community. Participant 1 stated: "I made many friends in church. I was deeply moved by their love, kindness, generosity. They were not like other Chinese. They had very happy family, they lived in peace, they loved people even strangers like me. I wanted to be this kind of person." Participant 2 talked about a Christian couple who shared their testimony about how their marriage was saved by their faith. She was deeply moved because her own marriage was about to break up at that time.

Inner hunger for unconditional love. Two female interviewees described their relationship with God as very intimate and reported complete satisfaction with their personal interaction with God. Conversely, they both indicated an insecure attachment bond with their mothers. Emotional dissatisfaction in their attachment with mother may contribute to their immersion in God's love. These two cases appear to support the compensatory model. Schore (2003) claims that insecure individuals use God and religion for interactive affect regulation more than secure individuals. However, Hall et al. (2009) argued that the internal working model of insecure individuals still influences their affect regulation, and IWM correspondence still operates simultaneously with "compensatory" affect regulation at an implicit level. These two participant cases appear to support this idea. Although these participants had reported an intimate relationship with God, they remained anxious and fearful of God, trying very hard to serve God and not sin against Him. For instance, Participant 2 stated:

I felt I was fulfilled with the Holy Spirit. I met Heavenly Father at one night. I saw Him. I was fulfilled by love. And God let me to see his love, and my own sins. I prayed, confessed my sins everyday. I thought God prepared that time for me.

"The end of man is the beginning of God." God serves as a safe haven and secure base for believers especially when Christians meet great trouble beyond their ability--their faith in God would be greatly enforced if their prayer is answered. Several participants described how they experienced God in the deep valley of their life and how, in the end, their faith was strengthened. Participant 5, for example, described:

To be honest, I have cheated on my husband before. But when I knew he was also cheating on me, my whole life collapsed. I was so desperate and depressed. If there is no God, I would be dead. I screamed in my heart for God's help. It was the weakest and darkest time of my life. I am so glad I still have Jesus. He saved me for the second time.

Participant 3 also explained:

When my second son brought the tragedy to me, I felt there was no way for us to live. I was on my knees praying many times. There was one time, thank God, He gave me a sentence: "A bruised reed He will not break. And smoking flax He will not quench." I relied on Him because of this sentence.

\section{Discussion}

\subsection{Traditionalism and Insecure Attachment}

The quantitative study showed that Chinese traditional values such as harmony, family orientation, face, and traditionalism were positively correlated with insecure parental attachment. What might this mean? There are two possible explanations. First, ECR-RS is not applicable in China due to cultural bias. For example, the avoidant dimension has six items which address verbal communication between parents and children.

1. It helps to turn to this person in times of need.

2. I usually discuss my problems and concerns with this person.

3. I talk things over with this person.

4. I find it easy to depend on this person.

5. I don't feel comfortable opening up to this person.

6. I prefer not to show this person how I feel deep down.

Based on observations from interviews, even participants with secure attachment do not talk about their feelings with parents; they do not discuss their problems with parents because they do not want parents to worry about them or are afraid of letting them down. It is not surprising that most of the secure attached participants could be categorized as avoidant attached. Just as addressed in the literature review section, the attachment theory and its categorization are developed in Western culture which encourage independence, uniqueness, verbal communication and emotional expressiveness. ECR as a typical measurement in Western culture might not be 
applicable for Chinese people who align with traditional interdependent cultural norms. In other words, there might be an overpathologizing tendency embedded within the instrument.

Secondly, there might be a possibility that Chinese traditional cultural values may be connected to insecure especially anxious parental attachment. That could also explain why self-orientation has negative association with insecure attachment.

Past research has consistently indicated that Chinese parents are more likely than North American parents to use physical coercion and verbal hostility in parent-child interactions (Leung, Lau, \& Lam, 1998; Steinberg et al., 1992). Xu et al. (2005) suggested that mainland Chinese mothers who adhered to Chinese traditional values of collectivism, conformity to norms, emotional self-control and humility were associated with an authoritarian parenting style. Authoritarian parenting might represent a way of training their children to fit within the traditional moral code of benevolence, righteousness, propriety, wisdom, and trustworthiness (ren, yi, li, zhi, xin in Mandarin). Based on those two possible explanations, we hypothesized that the intergenerational transmission of traditional values would affect Chinese parenting styles which would consequently contribute to the commonly found anxious attachment style. However, these hypotheses require further validation.

\subsection{Teng - The Implicit Love in Chinese Family}

Love is not a spoken word in Chinese family. Parents rarely verbalize love or kiss their children to express affection explicitly. They love children by 'xinteng' which is an indigenous word. Sundararajan (2005) translated 'xinteng' as heart-aching love, and she believes this to be the very core of Chinese attachment theory. She dissects this indigenous emotion into three interrelated frameworks: tender feelings in intimate relations, empathy toward others, and innate vulnerability to others. The sensitivity and responsiveness of Chinese mothers can not only detect but also anticipate the needs/stress in the child, protecting him/her from fear and providing emotional security. Japanese mothers also showed this tendency (Clancy, 1986). In China the ideal mother's vigilance and commitment to protection is manifested as worry (Sundararajan, 2005). Ancient texts also display this tendency: "Parents are anxious lest their children should be sick" (Confucian Analects, 1971, 2/6, p. 148). This study also showed that Chinese mothers are inclined to be anxious about children's health, behavioral performance, and future. In the eyes of their children, a Chinese mother's worry is an expression of love and care. So the child who is guai (obedient) aims to alleviate their mother's worry about them by listening to her teaching and concealing any negative feelings or information. One classical poem 'Hymn of the traveller' by poet Mengliao in Tang dynasty vividly depicted the image of Chinese mother.

Threads adeptly brandished by a loving mother, sewed into garments for a son so soon to depart.

Her sewing picks up pace as the date approaches, worries of belated return echoing in her heart.

Who dare claim that the green grass

might somehow repay the sun for its warm hearth?

When children grow up, they also have xinteng for their parents and love them back implicitly. This is demonstrated by giving their parents money, buying things for them, teaching them healthy lifestyles, etc. They care about their parents through action and underneath the implicit love is the same worry. This special emotion tightens the whole family, connecting parents and children no matter how old they become or how far they go.

\subsection{Love Oriented Guan vs. Coercive Control}

Previous research examined the Chinese parenting styles and identified the indigenous word guan as similar to a characteristics of authoritarian parenting. However, Chao $(1994,2001)$ differentiated guan from authoritarian. He believed guan to be a kind of parental control more indicative of parental involvement and concern as well as positive and beneficial to a child's development, especially academic achievement (Dornbusch et al., 1987). Chinese children might also perceive guan positively as happens with high parental expectations (Chao \& Sue, 1996; Day et al., 2003). This study also shows that guanjiao is an essential Chinese characteristic to parenting strategy. However, it is helpful to delineate 'love oriented' guan from coercive control.

This study revealed that love oriented guan is associated with secure/healthy parental attachment. Love oriented guanjiao is both child-centered and affectionate. Parents may use verbal teaching, behavioral correction, or tender punishment to guanjiao their children to meet their parents' expectation according to a certain moral standard. An involved responsible parent is expected to guan his/her child, and would be deemed unqualified otherwise. Ancient Chinese texts say 'To feed without teaching, is the father's fault. To teach without severity, is the teacher's laziness.' (Three Character Classic) However, just as much research has shown that, many Chinese 
parents use authoritarian parenting that is harmfully coercive to raise their children. Those parents might say they guan jiao their children, but this kind of guan is harsh and harmful to children's mental health and development. This type of parenting is physically coercive and psychologically controlling and is believed to be associated with low self-esteem, poor school performance and aggressive behaviors (Chang, Lansford, Schwartz, \& Farver, 2004; Chang, Schwartz, Dodge, \& McBride- Chang, 2003; Chen et al., 1997). This study showed that coercive, punitive parental control damaged children's emotional security, fostering doubt as to whether their parents really loved them. Two female interviewees shared their emotional distress with regards to their authoritarian mothers, connecting the negative outcome to the adverse parenting.

\subsection{Implications of Indigenous Parent-Child Relationship Styles on God Attachment}

Based on quantitative results and qualitative findings, the correspondence model appears to be supported. Secure parental attachment is associated with secure God attachment and a positive God image. According to Bowlby's theory, the way a person sees themselves and others, known as one's internal working model, influences behavioral, emotional and cognitive responses in social interactions across one's life span. For example, individuals raised by a sensitive mother may tend to consider God as trustworthy, present, and loving. Conversely, growing up in an unstable family with high controlling parents, he/she may be anxious about relationship with God, worrying about God's true love, and viewing God as judgmental. In other words, the interactions between Christians and God seemed to reflect the parent-child dynamics.

Chinese parents typically pay more attention to child's outer behaviors and performance rather than their inner feelings and emotional development. The moral values, rituals, social norms are very important in the process of guanjiao (training and teaching). Likewise, when the individuals convert to Christianity, righteous behaviors are seen as signs of maturity for their spiritual life. Love for God is shown just as love for their parents is shown (e.g., monetary donations to church, contribution to church service, obedience to God's teaching, etc.).

Interview results additionally support compensation model which theorizes that when someone is not securely attached to the caregiver or emotionally satisfied by the parents love, his/her deep hunger for unconditional love would consequently drive him/her to God. Past literature shows that individuals with histories of avoidant attachment were found to be more likely to have experienced a sudden religious conversion (Granqvist, 1998; Granqvist \& Hagekull, 1999; Kirkpartrick, 1999; Kirkpatrick \& Shaver, 1990). However, Macdonald et al. (2005) argued that even the motives to seek out and establish a relationship with God may have a compensatory goal, but once that relationship is established, the person's internal working model begins to assert themselves. The spiritual struggles of two female interviewees from the current study who are identified as insecurely attached seem to support this view. It does seem that underneath the compensatory intimate relationship with God, there is anxiety and uncertainty with regards to God's love.

\section{Limitation of the Present Study and Future Study Suggestion}

Compared to parents in big cities, those in villages expect that their sacrifice for their children will be rewarded in the future: "bring up children with the aim to guard against troubles in one's late years"( $\mathrm{Yu}, 2011)$. At the same time, they also expect children (sons) to bring honor to the family. Most of the participants from the present study are well educated and living in big cities and this population may be more affected by modern Western ideology. Therefore, it is suggested that future studies should include more rural areas and explore how the traditional culture may play a role in parenting, attachment, and spirituality. However, as discussed, Western measurement of attachment might not be suitable for Chinese population. The development and testing of an indigenous measurement is also needed for future study.

\section{Acknowledgement}

This research project is funded by Travis Research Institute, award Id: FP13XHH.

\section{References}

Agishtein, P., \& Brumbaugh, C. (2013). Cultural variation in adult attachment: The impact of ethnicity, collectivism, and country of origin. Journal of Social, Evolutionary, and Cultural Psychology, 7(4), 384-405. https://doi.org/10.1037/h0099181

Ainsworth, M. D. S., \& Bowlby, J. (1991). An ethological approach to personality development. American Psychologist, 46, 333-341. https://doi.org/10.1037/0003-066X.46.4.333

Ainsworth, M. D. S., Blehar, M. C., Waters, E., \& Wall, S. (1978). Patterns of attachment: A psychological study of the Strange Situation. Hillsdale, NJ: Erlbaum. 
Beck, R., \& McDonald, A. (2004). Attachment to God: The Attachment to God Inventory, tests of working model correspondence, and an exploration of faith group differences. Journal of Psychology and Theology, 32(2), 92-103. https://doi.org/10.1177/009164710403200202

Bowlby, J. (1969, 1982). Attachment and loss: Volume I attachment. New York: Basic Books.

Bowlby, J. (1973). Attachment and loss: Volume II separation anxiety and anger. New York: Basic Books.

Brennan, K. A., Clark, C. L., \& Shaver, P. R. (1998). Self-report measurement of adult attachment: An integrative overview.

Chang, L., Lansford, J. E., Schwartz, D., \& Farver, J. M. (2004). Marital quality, maternal depressed affect, harsh parenting, and child externalizing in Hong Kong Chinese families. International Journal of Behavioral Development, 28, 311-318. https://doi.org/10.1080/01650250344000523

Chang, L., Schwartz, D., Dodge, K., \& McBride-Chang (2003). Harsh parenting in relation to child emotion regulation and aggression. Journal of Family Psychology, 17, 598-606. https://doi.org/10.1037/0893-3200.17.4.598

Chao, R, K. (1994). Beyond parental control and authoritarian parenting style: Understanding Chinese parenting through the cultural notion of training. Child Development, 65, 1111-1119. https://doi.org/10.2307/1131308

Chao, R. K. (2001). Extending research on the consequences of parenting style for Chinese Americans and European Americans. Child Development, 72, 1832-1843. https://doi.org/10.1111/1467-8624.00381

Chao, R. K., \& Sue, S. (1996). Chinese parental influence and their children's school success: A paradox in the literature on parenting styles. Growing up the Chinese way: Chinese child and adolescent development, 93-120.

Chen, X., Dong, Q., \& Zhou, H. (1997). Authoritarian and authoritative parenting practices and social and school performance in Chinese children. International Journal of Behavioral Development, 21, 855-873. https://doi.org/10.1080/016502597384703

Chen, X., Liu, M., \& Li, D. (2000). Parental warmth, control, and indulgence and their relations to adjustment in Chinese children: A longitudinal study. Journal of Family Psychology, 14, 401-419. https://doi.org/10.1037/0893-3200.14.3.401

Chen-Bouck, L. (2016). The relations of Chinese mothers' endorsement of Chinese cultural values and parenting beliefs to their parenting styles and practices. Doctoral dissertation, University of Kansas.

Cheung, F. M., Leung, K., Fan, R. M., Song, W. Z., Zhang, J. X., \& Zhang, J. P. (1996). Development of the Chinese personality assessment inventory. Journal of Cross-Cultural Psychology, 27(2), 181-199. https://doi.org/10.1177/0022022196272003

Clancy, P. M. (1986). The acquisition of communicative style in Japanese. In Schieffelin, B. B., \& Ochs, E. (Eds.), Language Socialization Across Cultures (pp. 213-50).

Confucius, C. A. (1971). Great Learning and Doctrine of the Mean. Translated by James Legge.

Day, R. D., \& Lamb, M. E. (2003). Fathering in a Beijing, Chinese sample: Associations with boys' and girls' negative emotionality and aggression. In Conceptualizing and Measuring Father Involvement (pp. 182-210). Routledge.

Dornbusch, S. M., Ritter, P. L., Leiderman, P. H., Roberts, D. F., \& Fraleigh, M. J. (1987). The relation of parenting style to adolescent school performance. Child Development, 1244-1257. https://doi.org/10.2307/1130618

Dueck, A., \& Xu, H. (2017), Culture, attachment, and spirituality: Indigenous, ideological, and international perspectives. Research in the Social Scientific Study of Religion, 28, 255-277. https://doi.org/10.1163/9789004348936_013

Fraley, R. C., Heffernan, M. E., Vicary, A. M., \& Brumbaugh, C. C. (2011). The experiences in close relationships-relationship structures questionnaire: A method for assessing attachment orientations across relationships. Psychological Assessment, 23, 615-625. https://doi.org/10.1037/a0022898

Fraley, R. C., Waller, N. G., \& Brennan, K. A. (2000). An item-response theory analysis of self-report measures of adult attachment. Journal of Personality and Social Psychology, 78, 350-365. https://doi.org/10.1037/0022-3514.78.2.350 
Gaskins, S. (2013). The puzzle of attachment: Unscrambling maturation of and cultural contributions to the development of early emotional bonds. In Quinn, N., \& Mageo, J. M. (Eds.), Attachment reconsidered: Cultural perspectives on a western theory (pp. 33-66). Palgrave Macmillan. https://doi.org/10.1057/9781137386724_2

George, C., \& West, M. (1999). Developmental vs. social personality models of adult attachment and mental ill health. British Journal of Medical Psychology, 72, 285-303. https://doi.org/10.1348/000711299159998

Granqvist, P. (2002). Attachment and religiosity in adolescence: Cross-sectional and longitudinal evaluations. Personality and Social Psychology Bulletin, 28(2), 260-270. https://doi.org/10.1177/0146167202282011

Granqvist, P., \& Hagekull, B. (1999). Religiousness and perceived childhood attachment: Profiling socialized correspondence and emotional compensation. Journal for the Scientific Study of Religion, 38, 254-273. https://doi.org/10.2307/1387793

Granqvist, P., \& Kirkpatrick, L. (2013). Religion, spirituality and attachment. In K. I. Shin, H. (2009). Asian-American college students' parental attachment and their relationship with God. Christian Education Journal, 6(2), 353-375. https://doi.org/10.1037/14045-007

Harwood, R. L., Miller, J. G., \& Irizarry, N. L. (1995). Culture and attachment: Perceptions of the child in context. New York: Guilford Press.

Keller, H. (2008). Attachment-past and present. But what about the future? Integrative Psychological and Behavioral Science, 42, 406-415. https://doi.org/10.1007/s12124-008-9080-9

Kirkpatrick, L. A. (1999). Toward an evolutionary psychology of religion and personality. Journal of Personality, 67(6), 921-952. https://doi.org/10.1111/1467-6494.00078

Kirkpatrick, L. A., \& Shaver, P. R. (1990). Attachment theory and religion: Childhood attachments, religious beliefs, and conversion. Journal for the Scientific Study of Religion, 29, 315-334. https://doi.org/10.2307/1386461

Lawrence, R. T. (1991). The God image inventory: The development, validation and standardization of a psychometric instrument for research, pastoral and clinical use in measuring the image of God. Unpublished Doctoral Dissertation, The Catholic University of America, Washington D. C.

Lawrence, R. T. (1997). Measuring the image of God: The God image inventory and the God image scales. Journal of Psychology and Theology, 25(2), 214-226. https://doi.org/10.1177/009164719702500206

Leung, K., Lau, S., \& Lam, W. L. (1998). Parenting styles and academic achievement: A cross-cultural study. Merrill-Palmer Quarterly, 44, 157-172.

LeVine, R. A., \& Norman, K. (2001). The infant's acquisition of culture: Early attachment reexamined in anthropological perspective. In Moore, C. C., \& Mathews, H. F. (Eds.), The psychology of Cultural Experience (pp. 83-104). Cambridge, England: Cambridge University Press.

Markus, H. R., \& Kitayama, S. (1991). Culture and the self: Implications for cognition, emotion, and motivation. Psychological Review, 98, 224-253. https://doi.org/10.1037/0033-295X.98.2.224

McDonald, A., Beck, R., Allison, S., \& Norswortby, L. (2005). Attachment to God and parents: Testing the correspondence vs. compensation hypotheses. Journal of Psychology \& Christianity, 24(1), 21-28.

Mchale, J. P., Dinh, K. T., \& Rao, N. (2014). Understanding parenting and family systems among East and Southeast Asian-heritage families. In Selin, H. (Ed). Childrearing (163-173).

Otto, H., \& Keller, H. (2014). Different faces of attachment. Cambridge: Cambridge University Press. https://doi.org/10.1017/CBO9781139226684

Oyserman, D., Coon, H. M., \& Kemmelmeier, M. (2002). Rethinking individualism and collectivism: Evaluation of theoretical assumptions and meta-analyses. Psychological Bulletin, 128, 3-72. https://doi.org/10.1037/0033-2909.128.1.3

Quinn, N., \& Mageo, J. M. (2013b). Attachment and culture: An introduction. In Quinn, N., \& Mageo, J. M. (Eds.), Attachment reconsidered: Cultural perspectives on a Western theory (pp. 3-32). Palgrave Macmillan. https://doi.org/10.1057/9781137386724_1

Quinn, N., \& Mageo, J. M. (Eds.). (2013a). Attachment reconsidered: Cultural perspectives on a Western theory. Palgrave Macmillan. https://doi.org/10.1057/9781137386724 
Rothbaum, F., Morelli, G., \& Rusk, N. (2011). Attachment, learning, and coping: The interplay of cultural similarities and differences. In Gelfand, M. J., Chiu, C-y., \& Hong, Y-y. (Eds.), Advances in culture and psychology (pp. 153-216). Oxford: Oxford University Press.

Rothbaum, F., Weisz, J., Pott, M., Miyake, K., \& Morelli, G. (2000). Attachment and culture: Security in the United States and Japan. American Psychologist, 55, 1093-1104. https://doi.org/10.1037/0003-066X.55.10.1093

Schmitt, D. P., Alcalay, L., Allensworth, M., Allik, J., Ault, L., Austers, I., et al. (2004). Patterns and universals of adult romantic attachment across 62 cultural regions: Are models of self and of other pancultural constructs? Journal of Cross-Cultural Psychology, 35, 367-402. https://doi.org/10.1177/0022022104266105

Sorensen, N., \& Oyserman, D. (2010). Collectivism, Effects on Relationships. In Reis, H. T., \& Sprecher, S. K. (Eds.), Encyclopedia of Human Relationships (pp. 233-236). Thousand Oaks, CA: Sage.

Steinberg, L., Lamborn, S. D., Dornbusch, S. M., \& Darling, N. (1992). Impact of parenting practices on adolescent achievement: Authoritative parenting, school involvement, and encouragement to succeed. Child Development, 63(5), 1266-1281. https://doi.org/10.2307/1131532

Sundararajan, L. (2015). Understanding Emotion in Chinese Culture. Springer International Publishing. https://doi.org/10.1007/978-3-319-18221-6

Wang, C. C. D. C., \& Mallinckrodt, B. S. (2006). Differences between Taiwanese \& U.S. cultural beliefs about ideal adult attachment. Journal of Counseling Psychology, 53, 192-204. https://doi.org/10.1037/0022-0167.53.2.192

Wang, C., \& Song, Y. (2010). Adult attachment reconceptualized: A Chinese perspective, attachment: Expanding the cultural connections. New York: Routledge.

Xu, Y., Farver, J., Zhang Z., Zeng, Q., Yu, L., \& Cai, B. (2005). Mainland Chinese parenting styles and parent-child interaction. International Journal of Behavioral Development, 29(6), 524-531.

Yeo, J. (2010). The psychometric study of the attachment to God inventory and the brief religious coping scale in a Taiwanese Christian sample. Doctoral Thesis, Liberty University.

Yu, C-y. (2011). Analysis of the influencing factors of farmers' attitude towards the concept of "nurturing children and preventing old age" - Based on the survey data of more than 1,000 farmers in 10 provinces across the country. China Rural Observation, 3, 69-79.

\section{Copyrights}

Copyright for this article is retained by the author(s), with first publication rights granted to the journal.

This is an open-access article distributed under the terms and conditions of the Creative Commons Attribution license (http://creativecommons.org/licenses/by/4.0/). 